\title{
Building a Clinical Case Reports Database to Facilitate Case-Based Learning and Improve Critical Thinking of Medical Students
}

\author{
Jaroslav Majerník, Peter Jarčuška, Jozef Gonsorčík \\ Pavol Jozef Šafárik University in Košice, Faculty of Medicine, Trieda SNP 1, Košice, Slovakia \\ Email: jaroslav.majernik@upjs.sk, peter.jarcuska@upjs.sk,jozef.gonsorcik@upjs.sk
}

\begin{abstract}
This paper reports on development of our unified case reports database for medical students. The primary aim was to share professionals' clinical knowledge and skills through one of the most traditional medical experiences sharing form based on modern and anywhere available web services and technologies. Doing so, the clinical case reports that still play an invaluable role in case-based learning and in building of ability of students to think critically, can be used not only to learn and practice theoretical and practical consequences of clinicians' decisions, but also to prepare students for bedside teaching. To develop a generally acceptable system, we had to specify both the structure of education suitable materials and the platform for simple creation and sharing of such electronic outputs. As the result, the database of first clinical case reports was implemented into the information technologies infrastructure at Faculty of medicine in Kosice, Slovakia. It is offered to medical students in the form of web-based portal.
\end{abstract}

\section{INTRODUCTION}

$\mathrm{C}$ LINICAL experience represents a cornerstone of learning medical and health care practice which allows medical students to understand the high importance of interconnection between theoretical and practical knowledge. Various modern technologies based approaches, including ICT supported problem-based learning, virtual patients etc., were designed and integrated into the curricula of medical education providing institutions. The traditional paper-based patient case reports were often considered a part of an oldfashioned approach. However, they still remained an invaluable source of teaching materials. In particular, this is due to a real clinical background based on true patient stories and integration of professionals' skills and their experiences involved in best available strategies used to solve particular clinical case.

The most of medical education institutions are struggling to ensure a sufficient range of clinical placements for their students. These institutions recognize it is essential and irreplaceable component of medical education. Moreover,

This work was not supported by any organization the range of clinical learning hours can vary significantly from one country to another [1]. Except of transformation of theoretical knowledge into the practice, the students learn to face stressful situations when they are in clinical learning environment. On the other hand, the learners have to be prepared both the theoretically and pre-clinically before they will be allowed to enter the bedside teaching. Here, the clinical case reports play their education role even though they, as the sources of evidence, are located at the bottom parts of the evidence-based medicine hierarchy [2].

From the educational point of view, the case reports represent wealthy source of practical knowledge and experiences applicable in various medicine oriented pedagogical processes. Basically, the clinical case reports are presented in the form of clinical observations documentation that describe details of common and rare cases; characteristics of known and unknown diseases; novelty and/or new ideas in medicine; variations in diseases and their combinations; positive and negative effects of interventions; side effects of drugs usage as well as the ways professionals use to solve individual patient health related problems, respecting their best recent knowledge.

Because of the absence of general reporting guidelines designed for case reports, the clinicians and teachers report their clinical cases to the students in various heterogeneous forms using either electronic or paper-based approach. On the other hand, there are efforts of several medical associations to develop guidelines for clinical case reports in specific clinical domains. A positive example of such developments in this area is the initiative of international group of experts that developed Case Reports guidelines (CARE guidelines) [3, 4]. Aiming on reduction of bias, increasing of transparency and providing of first outputs of clinically applicable methods, these guidelines are useful mostly for publication of high quality clinical cases in scientific journals. However, many of the medical journals already stopped to publish clinical case reports as for the low citation index and thus negative effect on journal's impact factor [5]. In addition, the contemporary studies pointed out 
the case reports based on guidelines, whether modified or derived from CARE guidelines, have their usefulness and they are not rarely cited. Therefore, the well-written clinical case reports are again popularly published in newly established medical journals or in special supplements, volumes or websites of many prestigious publishers. From the educational point of view focused on higher education levels, the reporting of modern clinical cases tends to take a form of narrative stories that reveal gradually a solution of clinical problem $[6,7]$.

CARE guidelines have the potential to be a framework of clinical case reports for education purpose, but one has to be aware of broad variety of both general as well as specific objectives given in individual clinical disciplines that cannot result in a single case report framework applicable to all clinical cases and domains. As the evidence, various protocols and architectures of clinical case reports were published across different clinical disciplines $[8,9]$ or in biomedical applications $[10,11,12]$. This suggest the idea that the case reports significantly support clinical reasoning while forming an interface between medical knowledge and medical practice [13].

The inconsistency in case reports based education materials offered to medical students at our faculty forced us to initiate activities leading to the development of clinical case reports database and to the motivation of our academic clinicians to participate on it and to utilize it in education process. In contrast to the above-mentioned publishing phenomena of clinical case reports, and because of the educational background of our work, we faced an opposite role. Our main task was to design not discipline specific, but rather generalized case report framework to suit as many disciplines as possible while keeping authors a reasonable space for integration of their specifics that may vary from case to case. Also, this interdisciplinary acceptable framework should be offered to the teachers together with online available tools used to design and share individual elements of their clinical cases to their learners.

\section{II.MATERIALS AND METHODS}

Before the system of clinical case reports was developed and integrated into the teaching process, we had to solve both the methodological as well as the technological problems. The methodological problems were aimed to find a uniform framework of case reports that will fit to the most of the clinical disciplines taught at the faculty. To solve the technologies related problems we searched for the best way, which our teachers will use to create, to share and to maintain the database of clinical case reports and that will be available to learners anytime and anywhere. Also, this repository had to be developed in the way, which will serve medical students as additional source of clinically relevant study material and which should help them in better preparation for their later real clinical praxis.
Individual forms of case reports presented to our students by the teachers at individual clinical departments were analysed in order to identify the best consensus across various clinical disciplines. Not surprisingly, the clinical teachers used a broad scale of options, starting by reading the patient history, examination notes, laboratory test results etc. in paper based health records, through PowerPoint like presentations and ending with the study of the records and laboratory test results stored in clinicians' information systems. However, no one offered comprehensive and structured report prepared in compliance with CARE guidelines or similar ones. In addition, no of the case reports was available outside of the university network even in secured platforms. Personal discussions followed afterwards and the committee consisting of vice-deans and guarantors of particular clinical study fields reviewed and annotated importance and usefulness of individual sections in these forms of case reports. The conclusion was to use a generalized structure of education suitable case reports as it was presented in [14]. The proposed generalised structure of clinical case reports consists of following elements:

1) Title - topic of interest related to the patient, disease, symptoms, interventions etc.;

2) Author(s) details - author(s) name(s) and affiliation to department(s) of medical faculty;

3) Medical discipline(s) - assigned medical discipline(s) related to the problem to be solved;

4) Annotation image - image associated to the problem of presented case report;

5) Annotation - a brief introduction to the problem and/or short summary of the case report background;

6) Patient's history (anamnesis) - medical, social and family history, abuses, current health problems and symptoms etc.;

7) Laboratory tests results - clinical findings, results of relevant physical examinations, special laboratory tests etc.;

8) Imaging methods - imaging diagnostic methods where applicable, images from modalities used, description of findings and obtained results;

9) Diagnosis - main diagnosis specified according to ICD-10 and resulting from clinical findings and tests, used also to search for similar case reports published at faculty's clinical case reports database/portal;

10) Differential diagnosis - diagnostic reasoning with all considered diagnoses in patient assessment process;

11) Therapy - therapeutic interventions performed, important dates, treatment administration, prognostic details etc.;

12) Discussion and comments - results of the case, assessment of the results achieved, recent patient's status, strengths of the case, risks and limitations if any, recommendations of relevant literature;

13) Keywords - key elements of the case, used also to search for similar case reports published at faculty's clinical case reports database/portal and

14) Courses - a list of associated courses taught at the faculty in medical disciplines, used also to search for another 
education materials and articles related to the course(s) published at faculty's clinical case reports database/portal.

Bearing in mind all the recent possibilities of modern technologies and the fact that our teachers presented clinical cases to our students in various heterogeneous forms, including paper-based and oral presentations during face-toface teaching activities, we decided to focus their attention and pedagogical activities in this area to the one unified and online available place. Thanks to the MEFANET network and its ICT activities that already brought several interdisciplinary useful educational tools $[15,16]$, we found the solution of this problem in tools used within this educational network. In addition, we wanted to maintain the nature of traditional case reports and not to transform them into the simulation or standalone learning management systems as it was done for example in [17].

As the technological solution, we decided to use our local instance of MEFANET's portal platform to host the repository of our clinical case reports developed for various clinical disciplines. This decision resulted from great potential of the platform to manage multimedia content as well as to provide different user roles and access to the published materials. The advantages for teachers include the possibility to use generalized structure of case reports, minimal requirements on their technical skills and many others. On the other hand, the students can find everything in one system and they can study individual clinical cases, together with other types of study materials, wherever and whenever they need. Furthermore, using this way and depending on authors decision, the individual case reports can be exported to the MEFANET's Central Gate and thus shared to the learners studying at all medical faculties in Czech Republic and Slovakia.

\section{RESULTS}

To ensure as extensive impact of our work as possible, to start our project efficiently, to find the best consensus for all disciplines, to address the widest community of clinical teachers and finally to reach continuously growing number of clinical case reports, the management of the faculty organized a meeting with academic clinicians and teachers in September 2017. More than 60 clinicians took part in this meeting and they were informed about ideas and aims of that educational activity. Case Report framework was presented to the participants and consequently it was revised together with opened discussion to prove it is acceptable for the academics teaching medical students of our faculty. This also led to elimination of potential confusions and incompleteness of some parts or descriptions. Then, the participants were informed about the Portal of multimedia support in the education of clinical and health care disciplines at Faculty of Medicine at Pavol Jozef Safarik University in Kosice as well as about the ways used to publish clinical case reports on this Portal. The 14 above-mentioned sections of case report structure related to the portal presentation were approved.
The core idea of our initiative was accepted quite positively and most of the participants agreed to participate on first round of case reports development that was opened from 1st October 2017 to 31st December 2017. The first case reports were send to the redaction within few days after the meeting took place and once the post-meeting discussions were finished. As we expected, the majority of the case reports was prepared in internal medicine, surgery and dental medicine too. The academic clinicians generated several tenths of cases within this three-month period. 63 of them were finished, rearranged to fit the portal's framework and layout and shared to the students.

The list of annotations shared at the web-based portal is shown in Figure 1. The Figure 2 shows an example of open clinical case report with access to the multimedia content.

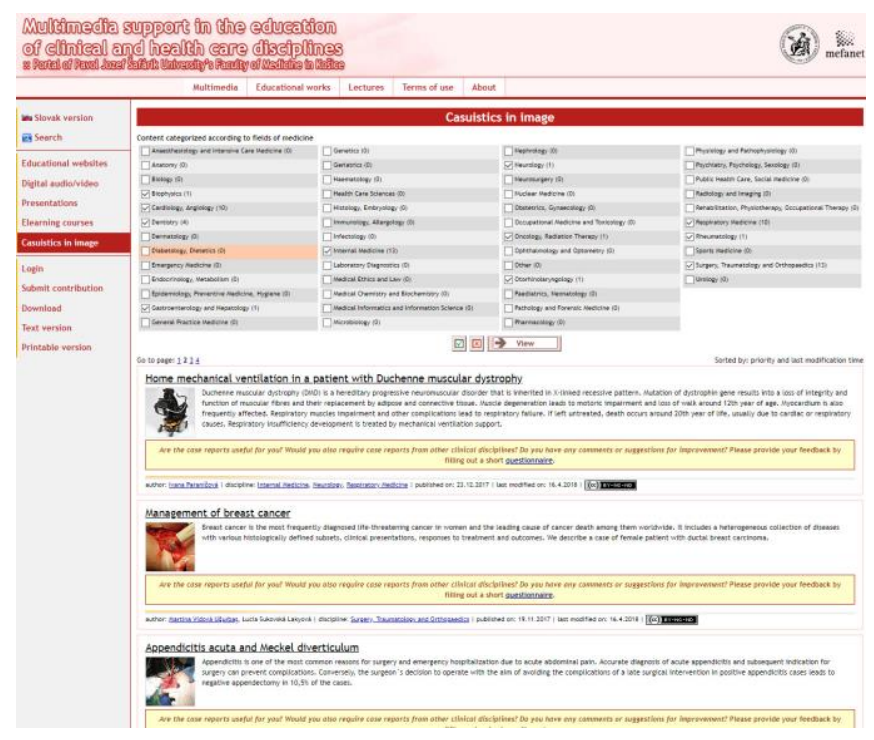

Fig. 1 The structure of annotations shared as clinical case reports database at the Portal of multimedia support in the education of clinical and health care disciplines at Faculty of Medicine at Pavol Jozef Safarik University in Kosice.

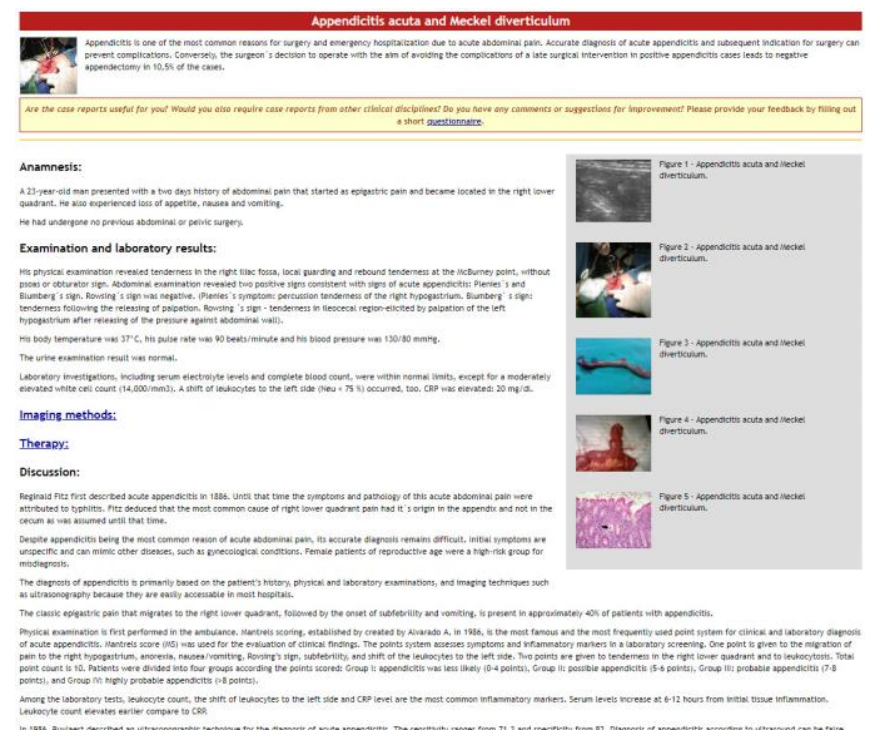

Fig. 2 An example of clinical case report published at faculty's portal. 
Then the activity to create clinical case reports database at our faculty continued and now the authors create their new and improve their existing cease reports depending on the most recent knowledge and students' feedback too.

There were totally 76 case reports finished and published at the Portal until now. All the case reports are published according to the portal's rules. To ensure the highest quality, guarantors of particular clinical disciplines review the content of all case reports. Then, each reviewed case report is labelled by the review logo to allow students recognizing reviewed and unreviewed clinical case reports. The reviewed case reports are automatically send to the Central Gate of MEFANET's portals, thus the students of all medical faculties in Czech republic and Slovakia can find them in one place and use them in their studies no matter which faculty they are from.

Another added value of our work, as we suppose, is that we expect increased motivation to create clinical case reports for education also in academic clinicians from another medical faculties involved in MEFANET. The motivation can be increased not only because of our pilot round of development, but maybe also because of the first positive students' feedbacks we noticed after publishing our pilot case reports.

\section{CONCLUSION}

Even if the scientific impact of clinical reports is considered as very low, we suggest the critical thinking of medical students can be improved thanks to the proper combination of high-quality clinical case reports, other traditional and electronic education methods used at the faculty and clinical bedside teaching. Except of others, the benefits for medical students included access to the valuable clinical experiences.

Medical education involves various teaching methods and strategies showing students clinical stories and examples based on real patients' health related problems. Even if the evidence-based medicine is considered to be one of the most relevant methods revealing background of systematic research and scientific clinical outputs, individual case reports have still a great potential to increase students' medical knowledge. Finally, we have to conclude the case reports offer students very challenging opportunities to evolve their clinical skills, but also to foster scientific and critical thinking.

\section{ACKNOWLEDGMENT}

Results presented in this work were obtained with the support of the national agency's grant KEGA 011UPJS$4 / 2019$ "Increasing of competences and critical thinking level in students of medical study programs using simulation tools of Problem-Based Learning and Evidence-Based Medicine".

\section{REFERENCES}

[1] Liu M., Chan Y.M., Tee S., Gu K., Luo Z.M., Wong T.K.S., "An iterative approach to enhance the clinical learning experience in Macao nursing education", International Journal of Nursing Sciences, 6, 2019, pp. 216-220, http://dx.doi.org/10.1016/j.ijnss.2019.01.005.

[2] van Haselen R.A., "Towards improving the reporting quality of clinical case reports in complementary medicine: Assessing and illustrating the need for guideline development", Complementary Therapies in Medicine, 23, 2015, pp. 141-148, http://dx.doi.org/10.1016/j.ctim.2015.01.009.

[3] CARE guidelines. [On-line] Available at WWW: <http://www.carestatement.org>

[4] Gagnier J.J., Kienle G., Altman D.G., Moher D., Sox H. Riley D., "The CARE Guidelines: Consensus-based Clinical Case Reporting Guideline Development", Global advances in health and medicine. 2(5), 2013, pp. 38-43.

[5] Firat A.C., Araz C., Kayhan Z.. "Case reports: Should we do away with them?", Journal of Clinical Anesthesia. 37, 2017, pp. 74-76, http://dx.doi.org/10.1016/j.jclinane.2016.10.006.

[6] Hurwitz B., "Narrative constructs in modern clinical case reporting", Studies in History and Philosophy of Science, 62, 2017, pp. 65-73, http://dx.doi.org/10.1016/j.shpsa.2017.03.004.

[7] Agha R.A., Fowler A.J., Saetta A., Barai I., Rajmohan S., Orgill D.P. "The SCARE Statement: Consensus-based surgical case report guidelines", International Journal of Surgery, 34, 2016, pp. 180-186, http://dx.doi.org/10.1016/j.ijsu.2016.08.014.

[8] Agha R.A., Farwana R., Borrelli M.R., Tickunas T., Kusu-Orkar T., Millip M.C., Thavayogan R., Garner J., Orgill D.P., "Impact of the SCARE guideline on the reporting of surgical case reports: A before and after study", International Journal of Surgery, 45, 2017, pp. 144148, http://dx.doi.org/10.1016/j.ijsu.2017.07.099.

[9] van Haselen R.A., "Homeopathic clinical case reports: Development of a supplement (HOM-CASE) to the CARE clinical case reporting guideline", Complementary Therapies in Medicine, 25, 2016, pp. 7885, http://dx.doi.org/10.1016/j.ctim.2015.12.019.

[10] Rajtúková V., Poláček I., Tóth T., Živčák J., Ižaríková G., Kovačevič M., Somoš A. Hudák R., "The manufacturing precision of dental crowns by two different methods is comparable", Lekar a Technika, 46(4), 2016, pp. 102-106.

[11] Kubicek J., Rehacek T., Penhaker M., Bryjova I., "Software simulation of CT reconstructions and artifacts", Lecture Notes of the Institute for Computer Sciences, Social-Informatics and Telecommunications Engineering, LNICST, Volume 165, 2016, Pages 428-437, http://dx.doi.org/10.1007/978-3-319-29236-6_41.

[12] Riley D.S. et al., "CARE guidelines for case reports: explanation and elaboration document", Journal of Clinical Epidemiology,89, 2017, pp. 218-235, http://dx.doi.org/10.1016/j.jclinepi.2017.04.026.

[13] Linsena A., Elshout G., Pols D., Zwaan L., Mamede S., "Education in Clinical Reasoning: An Experimental Study on Strategies to Foster Novice Medical Students' Engagement in Learning Activities", Health Professions Education, 4, 2018, pp. 86-96, http://dx.doi.org/10.1016/j.hpe.2017.03.003.

[14] Majernik J., Gonsorcik J. Repository of Clinical Case Reports. Mefanet 2017, Brno, 2017; 26-27.

[15] Schwarz D., Štourač P., Komenda M., Harazim H., Kosinová M., Gregor J., Hůlek R., Smékalová O., Křikava I., Štoudek R., Dušek L., "Interactive algorithms for teaching and learning acute medicine in the Network of Medical Faculties MEFANET", Journal of Medical Internet Research, 15 (7), 2013, pp. 135, http://dx.doi.org/10.2196/ jmir. 2590.

[16] Komenda M. et al., "Curriculum Mapping with Academic Analytics in Medical and Healthcare Education", PLoS ONE, 10 (12), 2015, e0143748, http://dx.doi.org/10.1371/journal.pone.0143748.

[17] Ali M. et al., "iCBLS: An interactive case-based learning system for medical education. International Journal of Medical Informatics", 109, 2018, pp. 55-69, http://dx.doi.org/10.1016/j.ijmedinf.2017. 11.004 . 